\title{
BMJ Open Efficacy and safety of furosemide for prevention of intradialytic hypotension in haemodialysis patients: protocol for a multicentre randomised controlled trial
}

\author{
Wenwen Chen (D) , ${ }^{1}$ Fang Wang, ${ }^{2}$ Yuliang Zhao, ${ }^{3}$ Ling Zhang (D) , ${ }^{3}$ Zhiwen Chen, ${ }^{2}$ \\ Mingjin Dai ${ }^{2}$
}

To cite: Chen W, Wang F, Zhao Y, et al. Efficacy and safety of furosemide for prevention of intradialytic hypotension in haemodialysis patients: protocol for a multicentre randomised controlled trial. BMJ Open 2021;11:e048015. doi:10.1136/ bmjopen-2020-048015

- Prepublication history for this paper is available online. To view these files, please visit the journal online (http://dx.doi org/10.1136/bmjopen-2020048015).

WC and FW contributed equally.

Received 18 December 2020 Accepted 08 June 2021

Check for updates

(c) Author(s) (or their employer(s)) 2021. Re-use permitted under CC BY-NC. No commercial re-use. See rights and permissions. Published by BMJ.

${ }^{1}$ Division of Nephrology, Kidney Research Institute, State Key Laboratory of Biotherapy and Cancer Center, Sichuan University West China Hospital, Chengdu, Sichuan, China ${ }^{2}$ Department of Nephrology, West China Hospital, Sichuan University/ West China School of Nursing, Sichuan University, Chengdu, Sichuan, China ${ }^{3}$ Department of Nephrology, Sichuan University West China Hospital, Chengdu, Sichuan, China

Correspondence to

Dr Ling Zhang;

zhangling_crrt@163.com

\section{ABSTRACT}

Introduction Intradialytic hypotension (IDH) is a frequent and serious complication of maintaining haemodialysis (HD) patients and associated with subsequent cardiovascular events and higher mortality. Furosemide is commonly used in non-dialysis chronic kidney disease patients and can effectively manage the volume and blood pressure. However, these agents are often discontinued on initiation of dialysis. Two large observational studies have demonstrated that furosemide can lower the rate of IDH episodes. However, there is still no randomised controlled trial (RCT) to investigate the efficacy and safety of furosemide for prevention of IDH in HD patients. The purpose of this study was to assess the efficacy of furosemide in reducing IDH in HD patients with residual renal function.

Methods and analysis A two-arm, parallel, multicente RCT will be conducted at 12 hospitals in China. An estimated sample of $560 \mathrm{HD}$ patients will be recruited. Eligible patients will be randomly assigned to treatment group (patients receive oral furosemide $80 \mathrm{mg} /$ day; after a 2-week treatment, if their urine volume is less than $400 \mathrm{~mL} /$ day, the dose of furosemide is adjusted to $160 \mathrm{mg} /$ day) and blank control group via a central randomisation system using 1:1 ratio. The primary outcome is the occurrence of IDH. Outcome assessors and data analysts will be blinded and participants will be asked not to reveal their allocation to assessors. The outcome analyses will be performed both on the intention-to-treat, which includes all patients randomised, and per-protocol population, which includes eligible patients who adhere to the planned treatment and follow-ups.

Ethics and dissemination The trial protocol has been approved by the Biomedical Research Ethics Committee of West China Hospital of Sichuan University (2019.385) Results will be presented at national and international conferences and published in peer-reviewed journals. Trial registration number ChiCTR2000039724.

\section{INTRODUCTION}

Intradialytic hypotension (IDH) is a common and serious complication of haemodialysis (HD) patients, with an incidence of up to $20 \%-30 \%$ of all dialysis sessions. ${ }^{12}$ The frequent occurrence of IDH increases the
Strengths and limitations of this study

- A multicentre randomised controlled trial of $560 \mathrm{pa}$ tients will be conducted to examine if furosemide can reduce intradialytic hypotension (IDH) and improve prognosis in haemodialysis patients.

- This trial will be conducted using rigorous methods, such as the use of a central computer-generated randomised sequence and the blinding of data analyst and outcome assessors.

- This trial will be administered a blank control, not a placebo control and dose contrast.

risk of thrombosis in vascular access, ${ }^{3}$ inadequate dialysis, ${ }^{4}$ cardiovascular disease $^{5}$ and mortality. ${ }^{367}$ An absolute nadir intradialytic systolic blood pressure (SBP) less than $90-100 \mathrm{~mm} \mathrm{Hg}$ was most potently associated with increase of about $30 \%$ mortality for patients with all range of predialysis blood pressure. $^{3}$

A series of factors may drive IDH through multiple mechanistic pathways. When the rapid removal of a large volume of blood water and solute overwhelmed normal physical compensatory mechanisms during dialysis therapy, the circulating volume and plasma refilling reduced, and followed, blood pressure. ${ }^{1}$ Based on the multifactorial pathophysiology of IDH, a number of strategies had been developed to reduce the frequency and severity of IDH, including the modulation of ultra-filtration (UF) ${ }^{8}$ qualitative changes in dialysate composition ${ }^{9}$ and lowering of dialysate temperature ${ }^{10}$ however, all of these had a limited effect. ${ }^{11}$ No other effective pharmacological approach is recommended to address IDH except adjusting antihypertensive drugs.

Furosemide, a common oral diuretic, is widely prescribed in chronic kidney disease (CKD) for managing volume and blood pressure. As a loop diuretic, furosemide increases 
the excretion of water, sodium and chlorine by inhibiting sodium and chloride reabsorption at the level of the thick ascending limb of the loop of Henle. ${ }^{12}$ Nonetheless, the role of diuretics in advanced CKD is not fully understood. Once patients start dialysis, it is often believed that dialysis can manage fluid overload and the use of diuretics is discontinued. Indeed for HD patients with residual renal function, diuretics can promote urine output, stabilise volume control, avoid ultrafiltration and thus reduce hypotension during dialysis. ${ }^{12}$

The effect of furosemide in dialysis has been investigated in several observational studies. Recently, one large observational study enrolling 12971 HD patients showed that loop diuretics can reduce dialysis hypotension significantly compared with control group (RR 0.95, 95\% CI, 0.92 to 0.99$).{ }^{13}$ These results were consistent with the previous Dialysis Outcome and Practice Pattern Study (DOOPS), which reported that diuretics reduce $45 \%$ hypotensive episodes and furosemide was the mostly widely used loop diuretic. ${ }^{14}$ Accumulating evidence showed that the use of loop diuretics can increase urine volume and sodium excretion, decrease the rate of weight gain in dialysis and reduce the risk of hospitalisation, ${ }^{13-15}$ while some other studies have shown that furosemide failed to delay kidney function decline or reduce mortality in dialysis patients. ${ }^{16}{ }^{17}$ Unfortunately, prospective randomised studies evaluating furosemide therapy for IDH are lacking.

In this study, we aim to conduct a prospective multicentre randomised controlled trial (RCT) to primarily examine if furosemide can reduce IDH in HD patients with residual renal function.

\section{METHODS AND ANALYSIS}

We followed the Standard Protocol Items: Recommendations for Interventional Trials statement for the reporting of our trial protocol. ${ }^{18}$

\section{Study design and setting}

A two-arm, parallel, blank-control, multicentre RCT evaluating the efficacy and safety of furosemide for prevention of IDH in patients will be conducted at West China Hospital of Sichuan University and other eleven dialysis centres in China. All the study centres can perform in-centre HD three times a week. We plan to recruit participants at each HD unit from June 2021 by the nephrologist and expect to end in March 2022. The eligible and consented patients will be randomly assigned to receive 6 months of furosemide treatment $(n=280)$ or blank control group $(n=280)$ at a 1:1 ratio. The cointervention between groups, including dialysis prescription and artificial kidney machines, will not be altered during the study period. All participants will be followed at least 1 year. The frequency of IDH episodes and other outcomes will be assessed at each HD session. This study has been approved by the biomedical research ethics committee, West China Hospital of Sichuan University
(2019.385). The study will be conducted in line with the declaration of Helsinki. A flow chart of the trial procedure is summarised in figure 1 .

\section{Participants}

All participants aged 18 years or older and diagnosed with end-stage renal disease according to the Kidney Disease: Improving Global Outcomes guideline ${ }^{19}$ at each HD unit will be assessed for eligibility. The inclusion criteria are as follows: participants who have been treated with HD three times a week for more than 3 months, with residual renal function of more than $200 \mathrm{~mL}$ urine output/day (based on a 24-hour urine collection, measured by a $200 \mathrm{~mL}$ measuring cup $)^{14}$ and consent to participate in the study.

Participants with SBP $\leq 100 \mathrm{~mm} \mathrm{Hg}$, a history of liver cirrhosis or concurrent liver impairment, experienced congestive heart failure (ejection fraction $\leq 50 \%$ ), acute myocardial infarction or stroke in the preceding 6 months, used any loop diuretics in the preceding 1 month, planned to receive a kidney transplant during the study period, or any other condition that the research staff judged as hard to complete or comply with or otherwise unsuitable for the study will be excluded.

The screening will be conducted by the research nephrologist through necessary physical examination and clinical tests. Eligible patients will be informed clearly of the aim and procedure of the research both verbally and via an information sheet. A consent form is needed to be completed and signed by each individual who agree to participate before enrolment. Information of all the patients will keep confidential and they will have the right to withdraw without prejudice at any time.

\section{Randomisation}

Eligible and consented patients will be randomly assigned to receive 6 months of furosemide treatment $(n=280)$ or blank control group $(n=280)$ at a 1:1 ratio via a central computer-generated randomised sequence. We will use a block randomisation in block sizes of 4 or 6 to keep balance between groups. The randomised sequence will be stored by the study coordinator and keep secret from the research nephrologist who recruits and evaluates the patients. Only after the informed consent is obtained, the allocation information will be provided to the patient's HD doctor, who will implement the intervention according to the study protocol. The study coordinator must ensure that the informed consent form has been obtained from each participant before randomisation to avoid selection bias.

\section{Interventions}

Following enrolment, participants will undergo a 1-week baseline period of comprehensive clinical assessment, including dry weight reduction, medication review and standardisation of their dialysis prescription. After randomisation, patients in both groups will receive HD three times a week over a 6 -month treatment period. The dialysis prescription, dialysate and artificial kidney machines will 


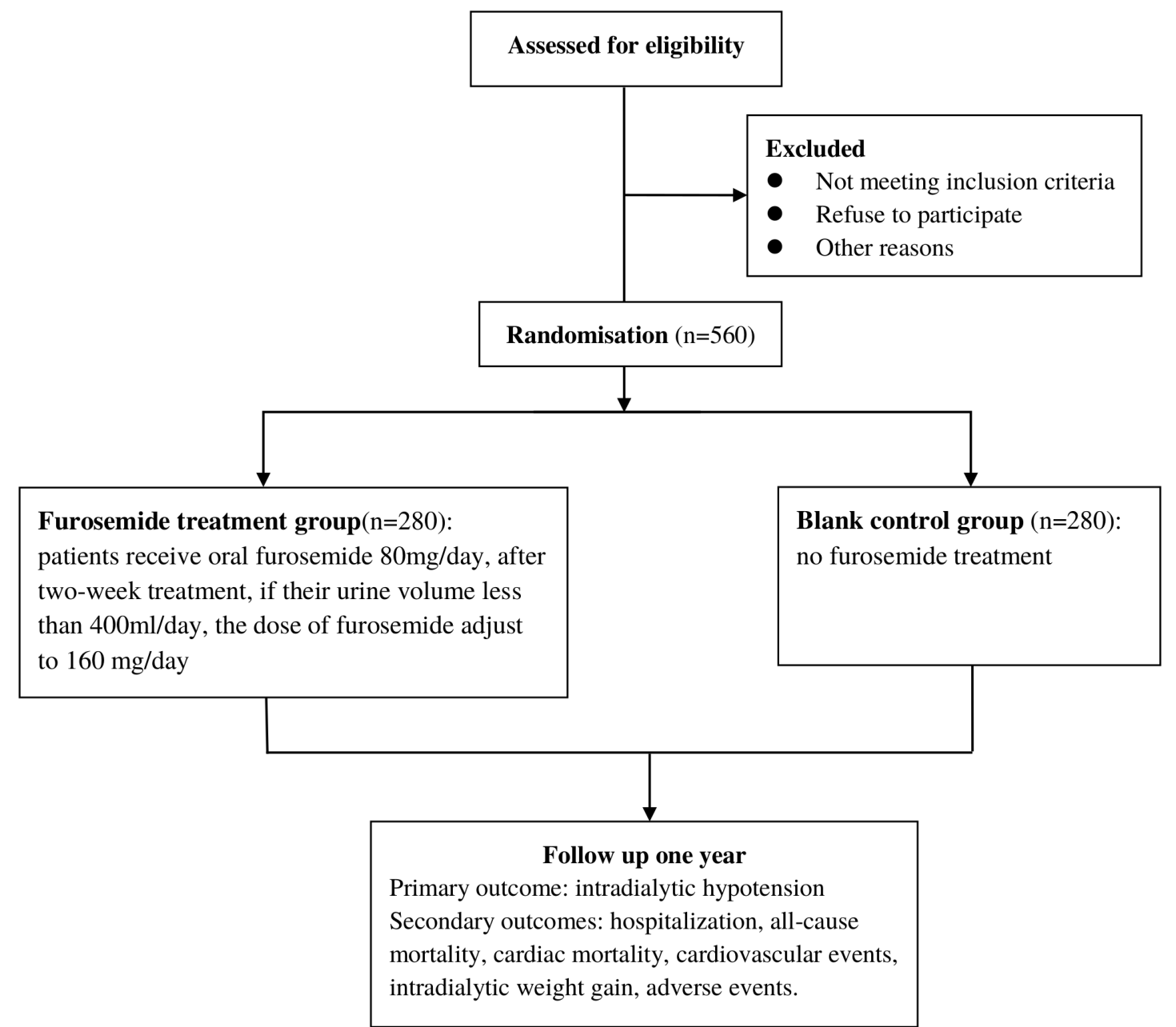

Figure 1 The summarised design of the trial.

not be altered during the treatment period. UF volumes will be adapted to reach $<5 \%$ of dry weight during each HD session. Dry weight will be determined clinically by the patient's attending nephrologist. The HD nurse assigned to each individual participant will record haemodynamic parameters, treatment parameters and IDH-related interventions on standard clinical HD run-sheets.

\section{Treatment group and blank group}

Eligible patients will be randomly assigned to treatment group or blank group. The patients in the treatment group will receive oral furosemide $80 \mathrm{mg}$ /day. After a 2-week treatment, if their urine volume was less than $400 \mathrm{~mL} /$ day, the dose of furosemide will be adjusted to $160 \mathrm{mg} /$ day. Furosemide treatment group will last for 6 months. Once the patient's daily urine output is less than $200 \mathrm{~mL}$, the use of furosemide will be discontinued. The patients in the blank group will receive no intervention, just as usual. Both groups will be followed up for 1 year.

\section{Primary outcome and definition of 'IDH'}

The primary outcome measures the rate of symptomatic IDH, and any IDH-related nursing interventions to treat hypotension episodes during each dialysis session. The definition of IDH is SBP $<90 \mathrm{~mm} \mathrm{Hg}$ (among patients with predialysis $\mathrm{SBP}<160 \mathrm{~mm} \mathrm{Hg}$ ) or $\mathrm{SBP}<100 \mathrm{~mm} \mathrm{Hg}$ (among patients with predialysis $\mathrm{BP} \geq 160 \mathrm{~mm} \mathrm{Hg}$ ). ${ }^{3}$ Blood pressure will be measured before dialysis, every 30 min during HD and after dialysis in each HD session of the study. IDH-related interventions will be defined as the use of the Trendelenburg position, manual reduction of UF rate, infusion of isotonic saline or hypertonic fluid, lowering of dialysate temperature or dialysis cessation. The number of symptomatic IDH episodes along with the duration of each dialysis treatment will be captured. The rate of IDH for each session will be calculated by dividing the number of episodes by the duration of the session in hours. Hypotensive events and symptoms (headache, cramps, nausea and vomiting) will be recorded and analysed as both the number of episodes and time of occurrence since the beginning of the HD session.

\section{Secondary outcomes}

The secondary outcomes are hospitalisation, all-cause mortality, cardiac mortality, cardiovascular events, interdialytic weight gain, dialysis symptoms and any adverse events. These data will be collected for all periods of the study. The outcome measurement time points are provided in detail in table 1 . 
Table 1 Timing of visits and data collection

\begin{tabular}{|c|c|c|c|c|c|}
\hline & \multirow[b]{2}{*}{$\begin{array}{l}\text { Screening } 1 \\
\text { week }\end{array}$} & \multirow[b]{2}{*}{$\begin{array}{l}\text { Baseline period } \\
0 \text { week }\end{array}$} & \multicolumn{3}{|c|}{ Treatment period } \\
\hline & & & 1 week & 6 months & $\begin{array}{l}\text { Follow-up period } \\
1 \text { year }\end{array}$ \\
\hline Eligibility & $X$ & & & & \\
\hline Informed consent & & $x$ & & & \\
\hline Type of vascular access & & $\mathrm{X}$ & & & \\
\hline Charlson comorbidity index score & & $x$ & & & \\
\hline Randomisation & & & $x$ & & \\
\hline \multicolumn{6}{|l|}{ Intervention } \\
\hline \multicolumn{6}{|l|}{ Outcomes } \\
\hline Hypotension episodes & & & $x$ & $x$ & \\
\hline Blood pressure & & & $x$ & $x$ & \\
\hline IDH-related nursing interventions & & & $x$ & $x$ & \\
\hline Dialysis symptoms & & & $x$ & $x$ & \\
\hline Interdialytic weight gain & & & $x$ & $x$ & \\
\hline Hospitalisation & & & & $x$ & $x$ \\
\hline All-cause mortality & & & & $x$ & $x$ \\
\hline
\end{tabular}

$\mathrm{IDH}$, intradialytic hypotension.

\section{Patient safety}

Any adverse events (described as unfavourable or unintended signs, symptoms or diseases occurring after treatment) related to furosemide therapy will be observed and reported by patients and practitioners during each dialysis session. In addition, all vital signs and adverse events will be measured and recorded at each dialysis session.

\section{Blinding}

Study investigators, nephrologists and participants will be aware of the treatment allocation. HD nurses, outcome assessors and data analysts will be blinded and participants will be asked not to reveal their allocation to assessors. In addition, we will blind interpretation of the study results to minimise misleading data interpretation. ${ }^{20}$

\section{Sample size determination}

Our primary study hypothesis is that furosemide treatment would achieve more reduction in the rate of IDH than the blank control group in HD patients. According to the previous study, the rate of IDH was 0.25 , compared with the control group, or that furosemide could reduce the rate of IDH was $0.55 .{ }^{14}$ Sample size calculations were conducted using G-Power V.3.1, with an $\alpha$ value of 0.05 and power of $80 \%$. The relative risk of IDH for treatment group relative to controls is 0.6 , and a sample size of 250 patients per arm is required. The dropout rate of furosemide treatment during the study was estimated to be $10 \%$, so a minimum sample size of 560 patients will be needed in each group.

\section{Monitoring}

We will develop an independent data monitoring committee (DMC), responsible for the monitoring the quality and regulatory compliance of the trial, as well as ensuring the safety of participating patients. The DMC will consist of six members with expertise in nephrology, 
dialysis management, dialysis care, trial methodology and biostatistics. The DMC meeting will be held once in 3 months; at the request of DMC, the meeting may take place every 2 months. We will develop a procedural document for the DMC meeting, and strictly follow the document.

\section{Patient and public involvement \\ No patient involved.}

\section{Statistical analysis}

Group comparisons will be undertaken using $\chi^{2}$ tests for categorical characteristics, and either analysis of t-test or Wilcoxon rank sum tests for continuous variables. The outcome analyses will be performed both on the intentionto-treat, which includes all patients randomised, and perprotocol population, which includes eligible patients who adhere to the planned treatment and follow-up.

\section{Ethics and dissemination}

The trial protocol has been approved by the Human Research Ethics Committee of West China Hospital of Sichuan University and other 11 dialysis centres in China. Participants were informed of the aims and nature of the research both verbally and via an information sheet. They were requested to complete an informed written consent before enrolment. Confidentiality of the information provided by the participants and their right to withdraw without prejudice are enforced throughout the study duration.

All individual data are securely stored, password protected and accessible by the research team only. The results of the trial will be presented at national and international scientific conferences and be submitted for publication in peer-reviewed journals.

\section{DISCUSSION}

This trial is expected to provide convincing evidence about the preventive effect of furosemide therapy for IDH. Although number of strategies have been developed to reduce the frequency and severity of IDH, all of those are difficult to conduct and hard to promote in clinical practices. To our knowledge, no effective pharmacological approach is recommended to address IDH except adjusting antihypertensive drugs.

Episodes of IDH can cause serious adverse events (renal ischaemia, cardiovascular disease and mortality), while the use of diuretics in HD patients has the potential to improve interdialytic fluid status. ${ }^{12}$ Diuretics are commonly prescribed for hypertension and volume management in CKD patients before dialysis. Currently, many clinicians discontinue these medications when individuals start dialysis, and diuretics are used infrequently in dialysis patients with residual renal function, studies in China report diuretic use between $6 \%$ and $29 \%^{21} 22$ and the dosing is also variable, with daily furosemide dose of $40-80 \mathrm{mg}$ reported in China. ${ }^{21} 22$ Continuation of loop diuretics after HD initiation was associated with lower rates of hospitalisation and IDH as well as lower interdialytic weight gain during the first year of dialysis. ${ }^{13}$ Based on published observational studies, the use of loop diuretics can increase urine volume and sodium excretion, decrease the rate of weight gain in dialysis and reduce the risk of hospitalisation. ${ }^{13-15}$ Among them, furosemide proved to be effective for prevention of IDH in HD patients. ${ }^{13-15}$ However, to our knowledge, prospective randomised studies evaluating furosemide therapy for IDH are lacking. Considering the heavy burden of IDH, there is a call for validation of the efficacy of this cheap and convenient strategy. Through our carefully designed RCT, we will offer trustworthy evidence for the effects of furosemide for IDH.

The major concern about the development of ototoxicity and other side effect of loop diuretics in dialysis patients hindered its use in HD. ${ }^{12}$ Another possible reasons for discontinuation of the use of loop diuretics include the assumption that dialysis treatment alone is sufficient for management of fluid overload and underestimation of its benefit for HD patients. A recent systematic review has found that loop diuretics may benefit HD patients by reducing the incidence rate of IDH, all-cause mortality and cardiovascular mortality for HD patients. ${ }^{23}$ However, evidence about its safety is still limited. The present prospective study will confirm the efficacy and gain evidence about the safety of furosemide in HD and further guide the clinical practices.

There are several limitations of this study. First, we did not administer a placebo control and dose contrast. Thus, the placebo effect may not be well parcelled out. However, it is difficult to get the placebo medicine, which is similar to furosemide in our study. Furosemide has some side effects, and blood pressure of patients vary, so it is not appropriate to administer dose contrast considering the limited sample sizes. For the safety of patients, the study gives a safe dose. Other assessments, including placebo and dose control with IDH, will be considered in the future study. Second, the definition of IDH is objective. In contrast, HD-related symptom frequency was much higher in a survey, ${ }^{24}$ but patients experience symptoms at varying thresholds of $\mathrm{BP}$ change and nadir, so it is difficult to recognise the symptom of IDH. However, hypotensive symptoms (headache, cramps, nausea and vomiting) will be recorded and analysed in our study. Our methods for recruitment, randomisation, allocation, outcome assessment and data collection methods have been carefully designed to minimise bias.

In summary, based on evidence from observational studies, furosemide seems to be a promising treatment for prevention of IDH in HD patients. Despite all the potential advantages of the use of diuretics in maintaining HD patients with residual renal function, furosemide is used infrequently in China and most countries. Our study will gain evidence from RCT and provide an effective way for reducing IDH in maintaining HD patients. Since we adopted a relatively low and safe dose of furosemide, more 
prospective studies investigating the ideal dose, especially dose based on the residual kidney function of individual are needed. Further studies are expected to offer trustworthy evidence for the effects of furosemide and stimulate furosemide to be better used in HD patients despite numerous potential benefits and few side effects.

In summary, we will conduct a definitive multicentre RCT to assess the efficacy and safety of furosemide for prevention of IDH in HD patients. We will use rigorous methods to minimise bias and set up several working committees to ensure quality conduct of the trial. Our study will gain evidence from RCT and provide an effective way for reducing IDH in maintaining HD patients. Further studies are expected to offer trustworthy evidence on the effects of furosemide and stimulate furosemide to be better used in HD patients despite numerous potential benefits and few side effects.

Contributors WC, FW, YZ and LZ conceived and designed the study. WC and FW drafted the manuscript. LZ, YZ, ZC and MD critically revised the manuscript. All authors have read and approved the manuscript.

Funding This study is supported by 1.3 .5 project for disciplines of excellenceClinical Research Incubation Project, West China Hospital, Sichuan University (2020HXFH014).

Competing interests None declared.

Patient and public involvement Patients and/or the public were involved in the design, or conduct, or reporting, or dissemination plans of this research. Refer to the Methods section for further details.

Patient consent for publication Parental/guardian consent obtained.

Provenance and peer review Not commissioned; externally peer-reviewed.

Open access This is an open access article distributed in accordance with the Creative Commons Attribution Non Commercial (CC BY-NC 4.0) license, which permits others to distribute, remix, adapt, build upon this work non-commercially, and license their derivative works on different terms, provided the original work is properly cited, appropriate credit is given, any changes made indicated, and the use is non-commercial. See: http://creativecommons.org/licenses/by-nc/4.0/.

\section{ORCID iDs}

Wenwen Chen http://orcid.org/0000-0002-2586-9234

Ling Zhang http://orcid.org/0000-0003-1155-4802

\section{REFERENCES}

1 Daugirdas JT. Pathophysiology of dialysis hypotension: an update. Am J Kidney Dis 2001;38:S11-17.

2 Ettema EM, Zittema D, Kuipers J, et al. Dialysis hypotension: a role for inadequate increase in arginine vasopressin levels? A systematic literature review and meta-analysis. Am J Nephrol 2014;39:100-9.

3 Flythe JE, Xue H, Lynch KE, et al. Association of mortality risk with various definitions of intradialytic hypotension. J Am Soc Nephrol 2015;26:724-34.
4 Ronco C, Brendolan A, Milan M, et al. Impact of biofeedbackinduced cardiovascular stability on hemodialysis tolerance and efficiency. Kidney Int 2000;58:800-8.

5 Stefánsson BV, Brunelli SM, Cabrera C, et al. Intradialytic hypotension and risk of cardiovascular disease. Clin J Am Soc Nephrol 2014;9:2124-32.

6 Shoji T, Tsubakihara Y, Fujii M, et al. Hemodialysis-Associated hypotension as an independent risk factor for two-year mortality in hemodialysis patients. Kidney Int 2004;66:1212-20.

7 Tislér A, Akócsi K, Borbás B, et al. The effect of frequent or occasional dialysis-associated hypotension on survival of patients on maintenance haemodialysis. Nephrol Dial Transplant 2003;18:2601-5.

8 Pirkle JL, Comeau ME, Langefeld CD, et al. Effects of weight-based ultrafiltration rate limits on intradialytic hypotension in hemodialysis. Hemodial Int 2018;22:270-8.

9 Vareesangthip K, Davenport A. Reducing the risk of intradialytic hypotension by altering the composition of the dialysate. Hemodial Int 2020;24:276-81.

10 van Olden RW, van Meyel JJ, Gerlag PG. Acute and long-term effects of therapy with high-dose furosemide in chronic hemodialysis patients. Am J Nephrol 1992;12:351-6.

11 Agarwal R. How can we prevent intradialytic hypotension? Curr Opin Nephrol Hypertens 2012;21:593-9.

12 Trinh E, Bargman JM. Are diuretics underutilized in dialysis patients? Semin Dial 2016;29:338-41.

13 Sibbel S, Walker AG, Colson C, et al. Association of continuation of loop diuretics at hemodialysis initiation with clinical outcomes. Clin J Am Soc Nephrol 2019;14:95-102.

14 Bragg-Gresham JL, Fissell RB, Mason NA, et al. Diuretic use, residual renal function, and mortality among hemodialysis patients in the dialysis outcomes and practice pattern study (DOPPS). Am J Kidney Dis 2007;49:426-31.

15 Lemes HP, Araujo S, Nascimento D, et al. Use of small doses of furosemide in chronic kidney disease patients with residual renal function undergoing hemodialysis. Clin Exp Nephrol 2011;15:554-9.

16 Flinn A, Ledger S, Blake P. Effectiveness of furosemide in patients on peritoneal dialysis. Cannt $J$ 2006;16:40-4.

17 Medcalf JF, Harris KP. Walls $\mathrm{J}$. Role of diuretics in the preservation of residual renal function in patients on continuous ambulatory peritoneal dialysis. Kidney Int 2001;59:1128-33.

18 Chan A-W, Tetzlaff JM, Altman DG, et al. Spirit 2013 statement: defining standard protocol items for clinical trials. Ann Intern Med 2013;158:200-7.

19 Stevens PE, Levin A, Kidney Disease: Improving Global Outcomes Chronic Kidney Disease Guideline Development Work Group Members. Evaluation and management of chronic kidney disease: synopsis of the kidney disease: improving global outcomes 2012 clinical practice guideline. Ann Intern Med 2013;158:825-30.

20 Järvinen TLN, Sihvonen R, Bhandari M, et al. Blinded interpretation of study results can feasibly and effectively diminish interpretation bias. J Clin Epidemiol 2014;67:769-72.

21 Guo Q, Yi C, Li J, et al. Prevalence and risk factors of fluid overload in southern Chinese continuous ambulatory peritoneal dialysis patients. PLoS One 2013;8:e53294.

22 Tan BK, Yu Z, Fang W, et al. Longitudinal bioimpedance vector plots add little value to fluid management of peritoneal dialysis patients. Kidney Int 2016;89:487-97.

23 Tang X, Chen L, Chen W, et al. Effects of diuretics on intradialytic hypotension in maintenance dialysis patients: a systematic review and meta-analysis. Int Urol Nephrol 2021. doi:10.1007/s11255-02102805-x. [Epub ahead of print: 06 Mar 2021].

24 Weisbord SD, Fried LF, Mor MK, et al. Renal provider recognition of symptoms in patients on maintenance hemodialysis. Clin J Am Soc Nephrol 2007;2:960-7. 